\title{
Acoustic Characterization of Fluorinert FC-43 Liquid with Helium Gas Bubbles: Numerical Experiments
}

\author{
Christian Vanhille, ${ }^{1}$ Cristian Pantea, ${ }^{2}$ and Dipen N. Sinha ${ }^{2}$ \\ ${ }^{1}$ Universidad Rey Juan Carlos, Tulipán s/n, Móstoles, 28933 Madrid, Spain \\ ${ }^{2}$ Los Alamos National Laboratory, Materials Physics and Applications, MS D429, Los Alamos, NM 87545, USA \\ Correspondence should be addressed to Christian Vanhille; christian.vanhille@urjc.es
}

Received 20 September 2016; Accepted 21 December 2016; Published 19 January 2017

Academic Editor: Tai Thai

Copyright (c) 2017 Christian Vanhille et al. This is an open access article distributed under the Creative Commons Attribution License, which permits unrestricted use, distribution, and reproduction in any medium, provided the original work is properly cited.

In this work, we define the acoustic characteristics of a biphasic fluid consisting of static helium gas bubbles in liquid Fluorinert FC43 and study the propagation of ultrasound of finite amplitudes in this medium. Very low sound speed and high sound attenuation are found, in addition to a particularly high acoustic nonlinear parameter. This result suggests the possibility of using this medium as a nonlinear enhancer in various applications. In particular, parametric generation of low ultrasonic frequencies is studied in a resonator cavity as a function of driving pressure showing high conversion efficiency. This work suggests that this medium could be used for applications such as parametric arrays, nondestructive testing, diagnostic medicine, sonochemistry, underwater acoustics, and ultrasonic imaging and to boost the shock formation in fluids.

\section{Introduction}

This paper deals with the propagation of ultrasound in a biphasic bubbly fluid comprised of Fluorinert FC-43 liquid and helium gas bubbles: (FC-43)-He. The primary objective of this paper is to conduct a theoretical study of this interesting medium to determine whether it is a good candidate for generating low ultrasonic frequency signal from the frequency mixing of two high frequency ultrasonic waves. Therefore, we first evaluate the acoustic parameters, such as sound speed, sound attenuation coefficient, compressibility coefficient, and acoustic nonlinear parameter of this bubbly fluid through numerical simulations. These parameters then allow us to determine the frequency mixing efficiency of this unique medium in creating low frequency ultrasonic waves using nonlinear parametric theory and numerical experiments.

Bubbles created by acoustic cavitation are employed in sonochemistry and other industrial processes, such as cleaning, mixing, and water treatment [1-6]. Sonoluminescence has been observed under some particular circumstances [7]. Bubbles are also used in ultrasonic diagnosis and therapy $[8,9]$.
Fluorinert FC-43 $\left(3 \mathrm{M}^{\mathrm{TM}}\right)$ was chosen for this study due to its low sound speed $(646 \mathrm{~m} / \mathrm{s}$ ) and relatively large (for a liquid) acoustic nonlinearity parameter $\beta(6.8-7.6)$ [10-13]. Fluorinert is an electrically insulating, stable hydrocarbonbased fluid used in various cooling applications of electronic components. It has several very useful properties, such as a very low viscosity, excellent chemical and thermal stability below its boiling point $\left(\sim 165^{\circ} \mathrm{C}\right)$, and compatibility with sensitive materials, and the liquid is practically nontoxic. The dielectric constant of Fluorinert is approximately 10 times than that of air, deeming it safe for nonlinear acoustics studies, which typically require high excitation power. Fluorinert FC-43 was used in the past to generate a collimated, narrow beam of low frequency $(15-120 \mathrm{kHz})$, using primary frequencies around $1 \mathrm{MHz}[11,14]$.

A mixture of Fluorinert and gas bubbles was chosen for the present study because of the well-known acoustic nonlinearity enhancement found in other bubbly fluids. For example, water's acoustic nonlinearity increases drastically when air bubbles are added to the liquid [15-19].

Helium (He-4) was chosen because of the lower acoustic attenuation coefficient of the resulting bubbly fluid as compared to the fluid mixture made of air bubbles (see Table 1). 
Helium gas also allows the generation of much smaller bubbles with ease than with air. The difference frequency signal that can be generated using this novel bubbly fluid through frequency mixing because of its enhanced acoustic nonlinearity is expected to be much stronger than that is possible with air bubbles. The combination of enhanced nonlinearity, low sound speed, and low acoustic attenuation can facilitate the generation of highly collimated low frequency ultrasonic beams $[11,14]$. It is worth stressing the potential for practical applications of this unique fluid medium that include parametric arrays, nondestructive testing, and diagnostic medicine $[1,2,6,9]$. The low sound speed of FC43 allows the development of smaller length devices as the wavelength is considerably shorter than in usual liquids used. For example, the wavelength in FC-43 is approximately 3 times shorter than in water.

For the reasons mentioned above, it is important to first obtain the acoustic characteristics of this novel bubbly fluid (FC-43)-He as no such data currently exist in the literature. In this paper, we present a theoretical approach to determine such characteristics of this bubbly fluid. Section 2 presents the mathematical model used to perform the theoretical analysis. In Section 3.1, the sound speed, attenuation coefficient, and compressibility coefficient of the bubbly liquid are determined. The nonlinear propagation of finite-amplitude ultrasound in the medium is presented in Section 3.2, and the evaluation of the acoustic nonlinear parameter of the medium that turns out to be very large is discussed in Section 3.3. This larger nonlinearity is employed to analyze the generation of the difference frequency component from frequency mixing in Section 3.4. Section 4 presents the conclusions of this work.

\section{Materials and Methods}

We consider the following differential system formed by the Rayleigh-Plesset and the wave equations, valid for the modeling of nonlinearly oscillating gas bubbles interacting in a liquid with a nonlinear ultrasonic field:

$$
\begin{aligned}
& \frac{\partial^{2} v}{\partial t^{2}}+\delta \omega_{0} \frac{\partial v}{\partial t}+\omega_{0}^{2} v=a v^{2}+b\left(2 v \frac{\partial^{2} v}{\partial t^{2}}+\left(\frac{\partial v}{\partial t}\right)^{2}\right) \\
&-\eta p, \quad 0 \leq x \leq L, 0<t<T, \\
& \frac{\partial^{2} p}{\partial x^{2}}-\frac{1}{c_{l}^{2}} \frac{\partial^{2} p}{\partial t^{2}}=-\rho_{l} N \frac{\partial^{2} v}{\partial t^{2}}, \\
& \quad 0<x<L, 0<t<T, \\
& p=\frac{\partial p}{\partial t}=0, \quad t=0,0<x \leq L, \\
& v=\frac{\partial v}{\partial t}=0, \quad t=0,0 \leq x \leq L, \\
& p(x=0)=p_{0} \sin (\omega t), \quad 0<t \leq T, \\
& p(x=L)=g_{L}(t), \quad 0<t \leq T,
\end{aligned}
$$

where $v$ and $p$ are the coupled bubble volume variation and acoustic pressure: both are functions of the one-dimensional
TABLE 1: Values of sound speed, attenuation coefficient, and compressibility coefficient of FC-43 liquid with helium gas bubbles (bold font values), of FC-43 liquid with air bubbles, and of water with air bubbles. Driving frequency is $f_{r}=20 \mathrm{kHz}$, bubble radius is $R_{0 r}=7 \mu \mathrm{m}$, and bubble density in the liquid is $N_{r}=1 \times 10^{11} / \mathrm{m}^{3}$.

\begin{tabular}{lccc}
\hline At $N_{r}-R_{0 r}-f_{r}$ & $c_{\mathrm{Bf}}(\mathrm{m} / \mathrm{s})$ & $\alpha_{\mathrm{Bf}}(/ \mathrm{m})$ & $\kappa_{\mathrm{Bf}}(/ \mathrm{Pa})$ \\
\hline FC-43 \& helium & $\mathbf{5 0 1 . 1 2}$ & $\mathbf{0 . 2 3}$ & $\mathbf{2 . 1 4} \times \mathbf{1 0}^{-\mathbf{9}}$ \\
FC-43 \& air & 488.3 & 0.29 & $2.26 \times 10^{-9}$ \\
Water \& air & 842.28 & 0.082 & $1.41 \times 10^{-9}$ \\
\hline
\end{tabular}

space coordinate $x$ and time $t . L$ is the spatial dimension of the study performed until the last instant $T$.

In the wave equation, $c_{l}$ is the small amplitude sound speed of liquid, $\rho_{l}$ is the equilibrium density of liquid, and $N$ is the bubble density in the liquid.

In the Rayleigh-Plesset equation, $\omega_{0}=\sqrt{3 \gamma_{g} p_{g} /\left(\rho_{l} R_{0}^{2}\right)}$ is the bubble resonance, in which $\gamma_{g}$ is the specific heat ratio of gas, $R_{0}$ is the initial radius of bubbles, and $p_{g}=\rho_{g} c_{g}^{2} / \gamma_{g}$ is the atmospheric pressure of gas, where $\rho_{g}$ and $c_{g}$ are the equilibrium density and small amplitude sound speed of gas, $\delta=4 \mu_{l} /\left(\omega_{0} R_{0}^{2}\right)$ is the viscous damping coefficient of the bubbly liquid, for which $\mu_{l}$ is the kinematic viscosity of the liquid, $a=\left(\gamma_{g}+1\right) \omega_{0}^{2} /\left(2 v_{0}\right), b=1 /\left(6 v_{0}\right)$, where $v_{0}$ is the initial volume of bubbles, and $\eta=4 \pi R_{0} / \rho_{l}$.

The source of frequency $f$, pulsation $\omega=2 \pi f$, and amplitude $p_{0}$ excites the fluid from $x=0$. The time-dependent function $g_{L}(t)$ describes the behavior of ultrasound at $x=$ $L$, depending on the kind of problem we solve (standing or propagating waves).

The system takes into account the nonlinearity, dispersion, and dissipation due to the gas bubbles in the liquid. The model is described in detail in $[15,16,18]$. Acoustic streaming, buoyancy, Bjerknes forces, and bubble collapse are not considered [19].

Two theoretical approaches are used here. The first approach corresponds to the application of an analytic perturbation method to the second order that allows us to calculate the sound speed, attenuation coefficient, and compressibility coefficient in the bubbly liquid and this is presented in Section 3.1. This approach is also described in detail in $[17,18]$. The second approach corresponds to the application of the Snow-Bl code $[15,20]$ that allows us to describe the propagation of nonlinear ultrasound in the bubbly liquid, by tracking variables $p$ and $v$ in time, and is discussed in Section 3.2. This is to evaluate the nonlinear parameter of the medium, presented in Section 3.3, and also to study the medium's capability to create a difference frequency component from two finite-amplitude signals by nonlinear mixing, discussed in Section 3.4. The numerical model is described in $[15,20]$.

\section{Results and Discussion}

This section presents the results obtained from applying the two approaches described in Section 2 to (FC-43)-He. 

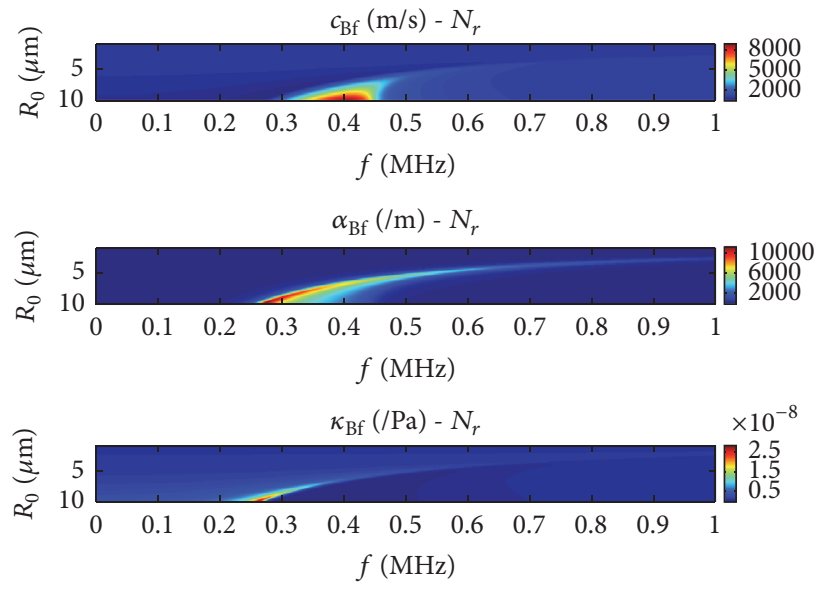

(a)
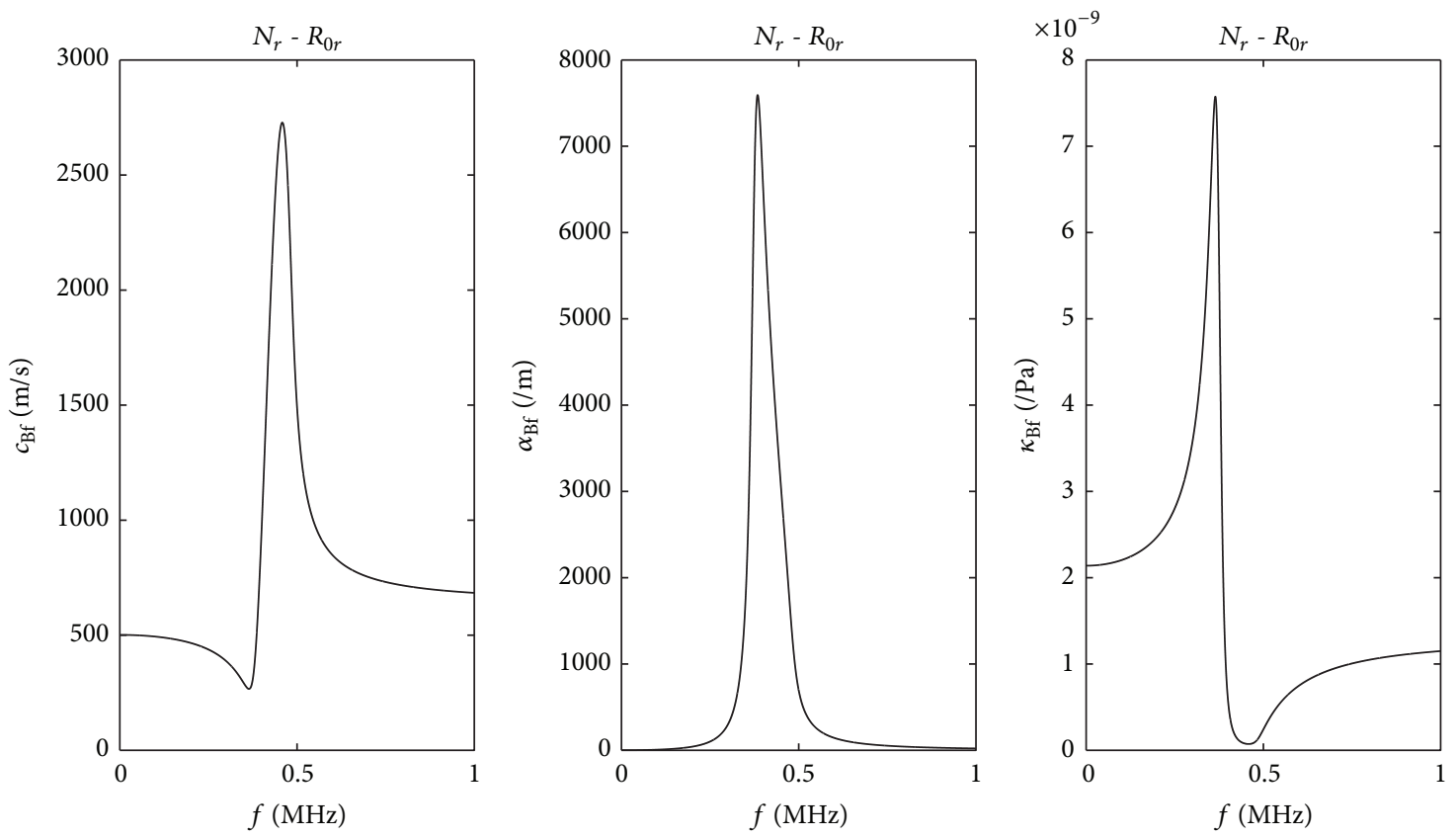

(b)

FIGURE 1: Sound speed, attenuation coefficient, and compressibility coefficient of the bubbly liquid (a) versus bubble size and driving frequency and (b) versus driving frequency for the specific bubble radius $R_{0 r}=7 \mu \mathrm{m}$. Bubble density is $N_{r}=1 \times 10^{11} / \mathrm{m}^{3}$.

These are presented in Section 3.1, and in Sections 3.2-3.4, respectively.

3.1. Acoustic Characteristics of the Bubbly Fluid. It is wellknown that the addition of even a relatively low concentration of gas bubbles with homogeneous density distribution into a liquid drastically modifies the acoustic properties of the liquid but hardly changes its density. In particular, the bubbly liquid becomes dispersive and its compressibility increases to a much higher value than for the homogeneous liquid in certain frequency ranges. In this section, we evaluate the sound speed $c_{\mathrm{Bf}}$, attenuation coefficient $\alpha_{\mathrm{Bf}}$, and compressibility coefficient $\kappa_{\mathrm{Bf}}$ of (FC-43)-He mixture, for several bubble sizes and over a large frequency range by conserving the same bubble density in the liquid $\left(N_{r}=1 \times 10^{11} / \mathrm{m}^{3}\right)$ (Figure 1(a)). In particular, we show the values for a specific bubble radius $\left(R_{0 r}=7 \mu \mathrm{m}\right)$ (Figure $1(\mathrm{~b})$ ) and for a specific frequency $\left(f_{r}=20 \mathrm{kHz}\right)$ (Table 1 , bold font values). These specific values will be used as reference values in the rest of the paper. The evaluation of the parameters follows the first approach described in Section $2[17,18]$. Table 1 also lists the corresponding results for Fluorinert FC-43 liquid with air bubbles and for water with air bubbles. The parameters used in this paper are the following: sound speed, density, and kinematic viscosity of Fluorinert FC-43 liquid as $c_{l}=646 \mathrm{~m} / \mathrm{s}$, $\rho_{l}=1860 \mathrm{~kg} / \mathrm{m}^{3}$, and $\mu_{l}=2.5 \times 10^{-6} \mathrm{~m}^{2} / \mathrm{s}$ and sound speed, density, and specific heat ratio of gas helium as $c_{g}=1016 \mathrm{~m} / \mathrm{s}$, $\rho_{g}=0.1637 \mathrm{~kg} / \mathrm{m}^{3}$, and $\gamma_{g}=5 / 3$, respectively. 

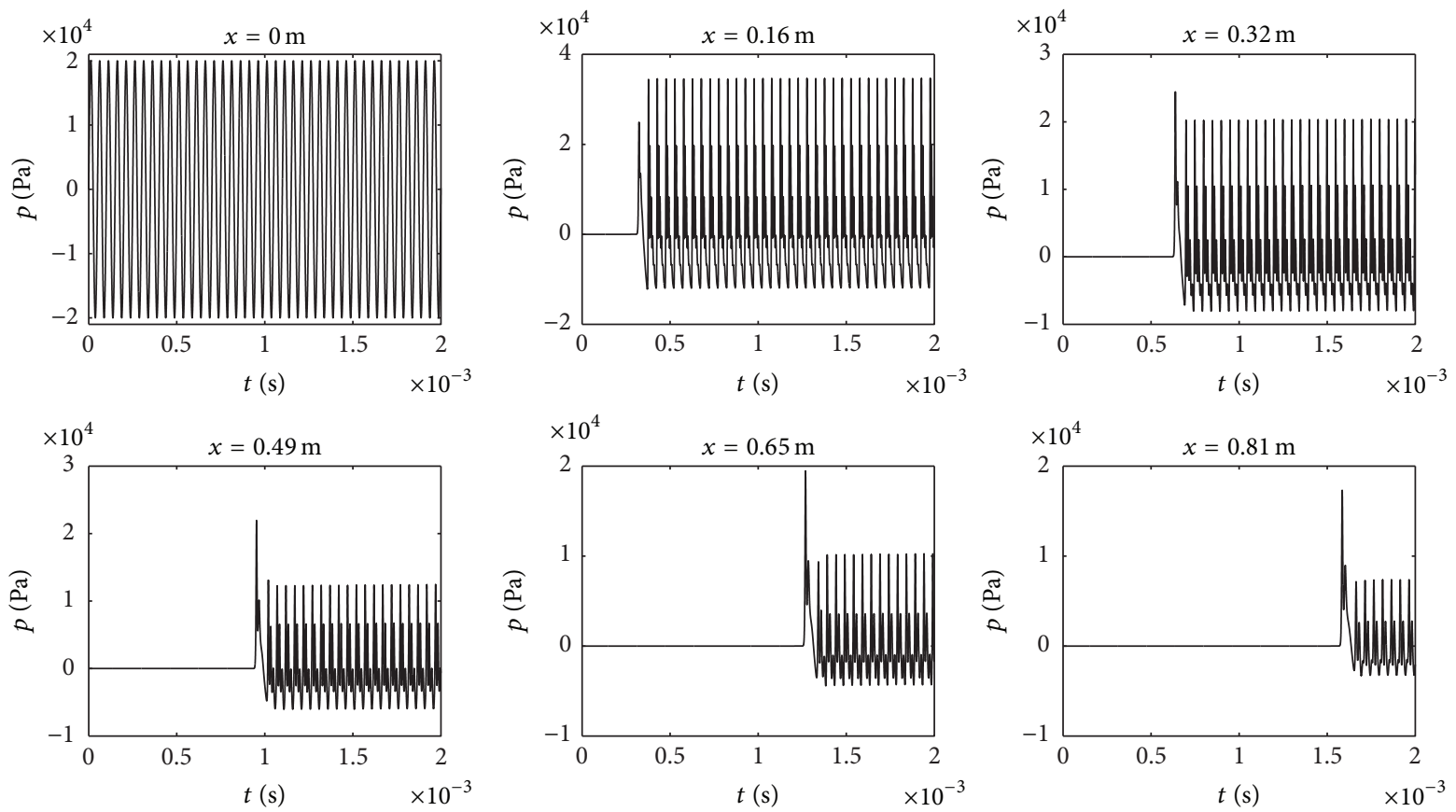

(a)
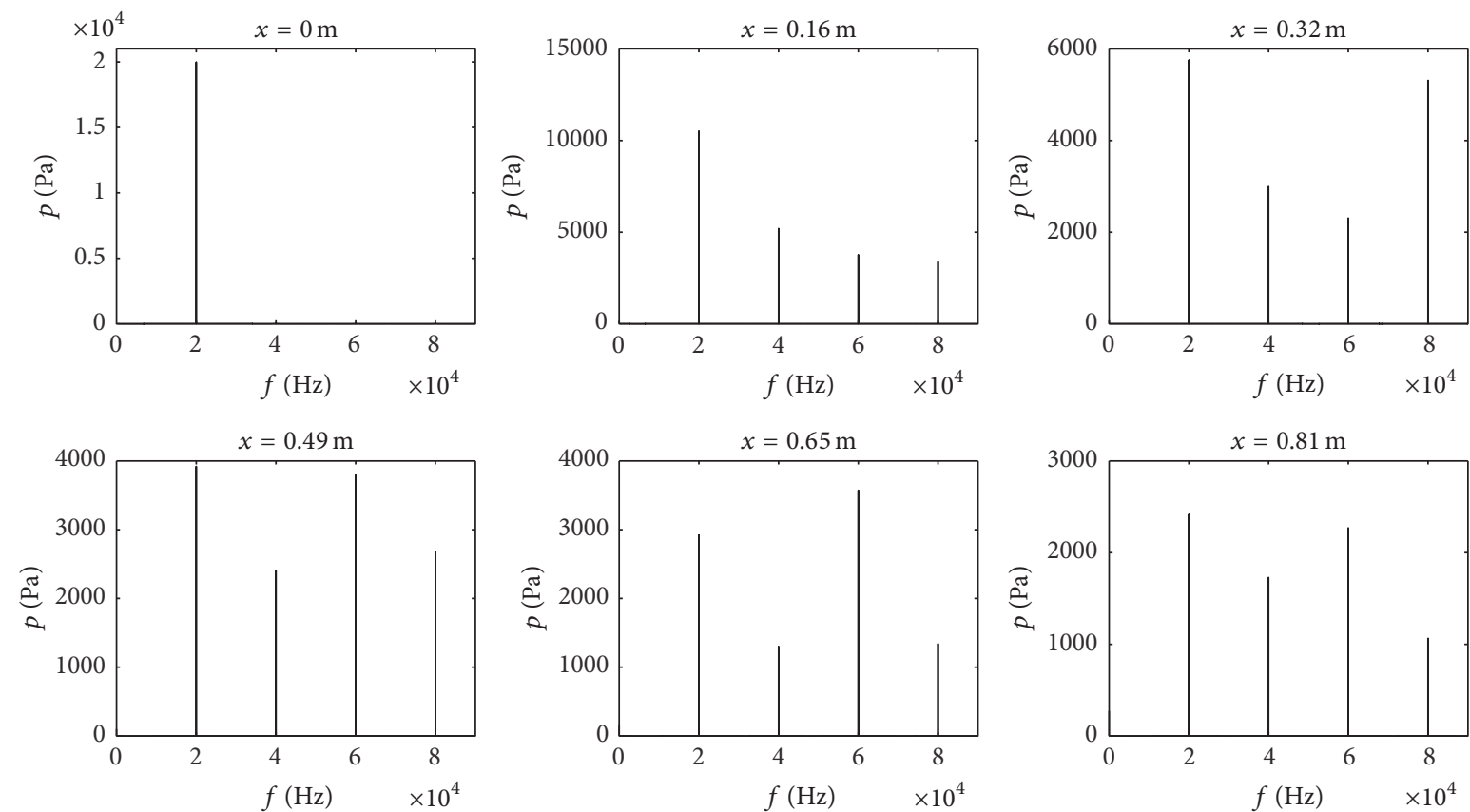

(b)

Figure 2: (a) Pressure waveform and (b) frequency content at several distances from the source. Bubble radius is $R_{0 r}=7 \mu \mathrm{m}$, bubble density is $N_{r}=1 \times 10^{11} / \mathrm{m}^{3}$, driving frequency is $f_{r}=20 \mathrm{kHz}$, and pressure amplitude at the source is $p_{0}=20 \mathrm{kPa}$.

It can be seen in Figure 1 that $c_{\mathrm{Bf}}, \alpha_{\mathrm{Bf}}$, and $\kappa_{\mathrm{Bf}}$ are frequency dependent. This dispersive nature affects the nonlinear ultrasonic field since each of its harmonic components travels at its own sound speed with its associated attenuation. It can be seen that at a frequency $f_{r}=20 \mathrm{kHz}$, situated well below the resonance frequency of the bubbles present, the frequency range of largest attenuation in the system under consideration can be avoided. This frequency corresponds to a low sound speed and high compressibility of the medium and this combination enhances its nonlinearity [10, 17-19].

3.2. Ultrasonic Propagation in the Bubbly Fluid. The compressibility change obtained by adding helium gas bubbles to Fluorinert FC-43 liquid (Figure 1 and Table 1) implies 


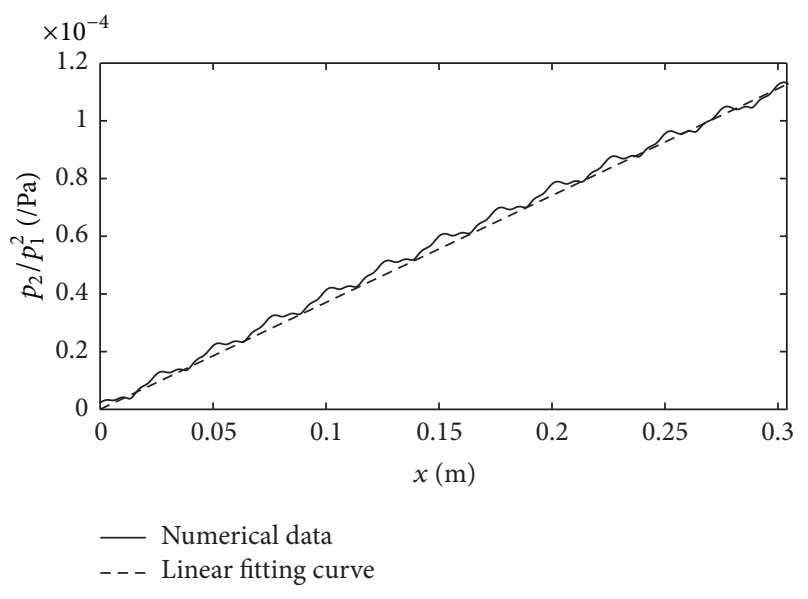

FIGURE 3: Second harmonic of pressure as a function of the square of the fundamental versus distance from the source. Bubble radius is $R_{0 r}=7 \mu \mathrm{m}$, bubble density is $N_{r}=1 \times 10^{11} / \mathrm{m}^{3}$, driving frequency is $f_{r}=20 \mathrm{kHz}$, and pressure amplitude at the source is $p_{0}=1800 \mathrm{~Pa}$.

a higher nonlinearity of the medium at certain frequency ranges [17-19]. This modification affects the behavior of ultrasound propagation through the bubbly liquid. The simulation of the nonlinear propagation of ultrasound in (FC43)-He is performed here by the second approach described in Section $2[15,20]$. In this section, we calculate the response obtained in the medium for bubbles of radius $R_{0 r}$ that are evenly distributed in the liquid at density $N_{r}$ for a continuous ultrasonic signal of amplitude $20 \mathrm{kPa}$ driven at $f_{r}$. Figure 2 shows the pressure waveforms obtained at several distances $x$ from the source with the corresponding frequency content.

As the wave travels through the bubbly liquid, the strong attenuation due to the bubbles reduces the amplitude and the signal undergoes a strong nonlinear distortion that is made evident by the asymmetry between compression and rarefaction amplitudes and by the creation of higher harmonic components.

3.3. Determination of the Nonlinear Parameter of the Bubbly Fluid. The numerical results presented in the previous section revealed the strong nonlinear character of ultrasound at quite high amplitude in (FC-43)-He mixture. In this section, we evaluate the nonlinear parameter $\beta$ of this bubbly liquid by applying the second approach described in Section $2[15$, 20]. The acoustic nonlinear parameter, $\beta=1+B /(2 A)$, measures the capacity of a medium to distort nonlinearly an acoustic wave, where $B / A$ is a measure of the nonlinearity in the equation of state of a material $[19,21-24]$. To this purpose, we use the finite-amplitude technique described in [25]. The amplitude values of the fundamental and second harmonic are calculated in the configuration shown in Section 3.2 (Figure 2) but for a lower amplitude at the source $(1800 \mathrm{~Pa})$. This is to avoid the formation of higher harmonic components (from third to higher) that can adversely affect the application of this technique. Figure 3 shows the second harmonic, $p_{2}$, as a function of the square of the fundamental, $p_{1}$, up to a distance of $x=30.4 \mathrm{~cm}$ from the source, as

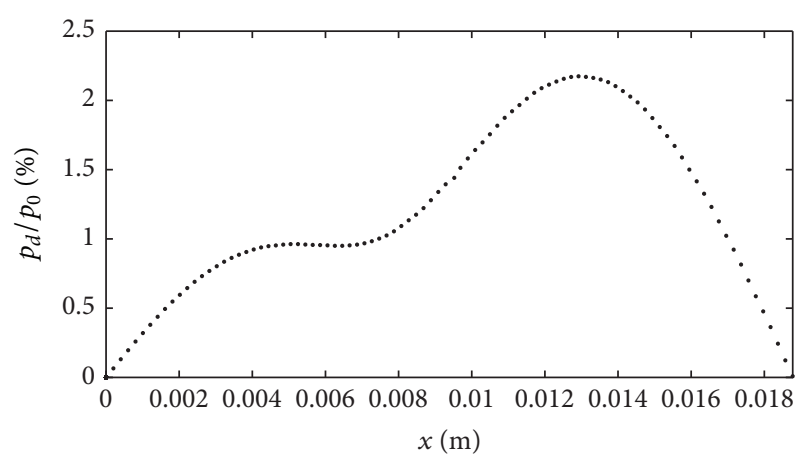

(a)

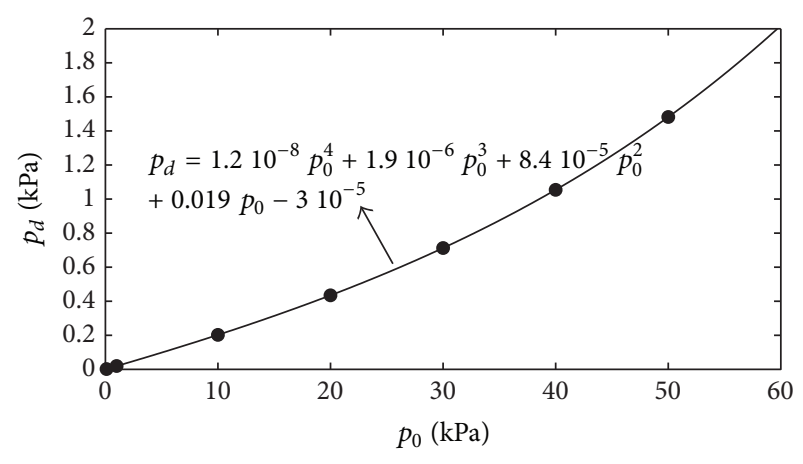

- $p_{d}$ Fitting curve

(b)

FIgURE 4: (a) Ratio of $p_{d}$ to $p_{0}$ (\%) versus distance from the source in the resonator and (b) maximal value of $p_{d}$ in the resonator versus $p_{0}$ (in $\mathrm{kPa}$ ). Bubble radius is $R_{0 r}=7 \mu \mathrm{m}$, bubble density is $N_{r}=1 \times$ $10^{11} / \mathrm{m}^{3}$, primary frequencies are $f_{1}=800 \mathrm{kHz}$ and $f_{2}=820 \mathrm{kHz}$, and difference frequency is $f_{d}=20 \mathrm{kHz}$. Pressure amplitude at the source is $p_{0}=20 \mathrm{kPa}$ in (a) and is varied up to $50 \mathrm{kPa}$ in (b).

well as a linear fit of the data. The slope of the linear fit is $S=3.71 \times 10^{-4} / \mathrm{mPa}$. The nonlinear parameter $\beta$ is defined by $\beta=S \rho_{\mathrm{Bf}} c_{\mathrm{Bf}}^{3} / \pi f$ [25-27], where $\rho_{\mathrm{Bf}}$ is the density of the bubbly liquid and $c_{\mathrm{Bf}}$ is the sound speed in the bubbly liquid at frequency $f$. The $\beta$ value is thus 1380 . This nonlinear parameter value is several orders of magnitude higher than the $\beta$ value measured in homogeneous Fluorinert FC-43 liquid, which is 7.6 [11].

3.4. Difference Frequency Generation in the Bubbly Fluid. The acoustic nonlinearity of the bubbly medium (FC-43)-He reflected by the very high nonlinear parameter $\beta$ obtained in Section 3.3 leads to the prospect of using this medium for generating a low difference frequency signal from two high frequency signals by nonlinear mixing $[11,14,28]$. A cavity filled with the bubbly medium (FC-43)-He is considered. Here, the second approach described in Section 2 is used [29]. The difference frequency $f_{d}$ is chosen to be $f_{r}$ by setting the primary frequencies at the source to $f_{1}=800 \mathrm{kHz}$ and $f_{2}=820 \mathrm{kHz}$. The length of the cavity is set to fit (3/4)th of the wavelength at $f_{d}$ in the bubbly liquid. The pressure amplitude at the source $p_{0}$ is set at $20 \mathrm{kPa}$. Figure 4 (a) displays the ratio 
of the difference frequency amplitude $p_{d}$ to $p_{0}$ expressed in percent versus distance $x$ from the source in the resonator. Figure 4(b) represents the maximal value of $p_{d}$ found in the resonator when $p_{0}$, expressed in $\mathrm{kPa}$, is varied from very small values up to $50 \mathrm{kPa}$.

Figure 4(a) shows a nonhomogeneous distribution in the cavity in terms of amplitude of difference frequency and shows that the point of maximal amplitude is located at $13 \mathrm{~mm}$ from the source. Figure 4(b) indicates that at low amplitude no difference frequency is present in the cavity. The difference frequency component is due to the nonlinearity of the medium that is decisive at finite-amplitude pressures. This means that the difference frequency amplitude increases with pressure amplitude at the source. The fitting curve of this component indicates that the response of the system follows a fourth-degree polynomial curve.

\section{Conclusions}

This work shows the high acoustic nonlinearity of a unique medium composed of Fluorinert FC-43 liquid in which a population of tiny helium gas bubbles is evenly distributed. The medium is characterized acoustically by evaluating the sound speed, attenuation parameter, compressibility coefficient, and nonlinear parameter. The effects of this medium on the propagation of ultrasound are presented, as well as its capability for generating difference frequency components. This work suggests the potential use of Fluorinert FC-43 liquid with helium gas bubbles as a nonlinear enhancer in various practical applications, including shock formation in fluids.

\section{Competing Interests}

The authors declare that there is no conflict of interests regarding the publication of this paper.

\section{Acknowledgments}

Christian Vanhille is deeply grateful to Dr. Cleofé CamposPozuelo. This work is funded by the Ministry of Economy and Competitiveness of Spain via the Research Project DPI201234613 and by the Los Alamos National Laboratory (Program Code 3N010A-DG08).

\section{References}

[1] F. R. Young, Cavitation, McGraw-Hill, London, UK, 1989.

[2] T. J. Mason and J. P. Lorimer, Applied Sonochemistry: The Uses of Power Ultrasound in Chemistry and Processing, Wiley-VCH, Weinheim, Germany, 2002.

[3] K. Yasui, T. Tuziuti, M. Sivakumar, and Y. Iida, "Theoretical study of single-bubble sonochemistry," Journal of Chemical Physics, vol. 122, no. 22, Article ID 224706, 2005.

[4] H. Delmas and L. Barthe, "Ultrasound mixing, homogenization, and emulsification in food processing and other applications," in Power Ultrasonics: Applications of High-Intensity Ultrasound, J. A. Gallego-Juárez and K. Graff, Eds., vol. 66 of
Woodhead Publishing Series in Electronic and Optical materials, pp. 757-791, Elsevier, Amsterdam, The Netherlands, 2015.

[5] C. Pétrier, "The use of power ultrasound for water treatment," in Power Ultrasonics: Applications of High-Intensity Ultrasound, J. A. Gallego-Juárez and K. Graff, Eds., vol. 66 of Woodhead Publishing Series in Electronic and Optical Materials, pp. 939972, Elsevier, Amsterdam, The Netherlands, 2015.

[6] O. V. Abramov, High-Intensity Ultrasound: Theory and Industrial Applications, Gordon and Breach, London, UK, 1998.

[7] W. Lauterborn, "Nonlinear acoustics and acoustic chaos," in Sound-Flow Interactions, vol. 586 of Lecture Notes in Physics, pp. 265-284, Springer, Berlin, Germany, 2002.

[8] F. Gao, C. Xiong, and Y. Xiong, "Constrained oscillation of a bubble subjected to shock wave in microvessel," Progress in Natural Science, vol. 19, no. 9, pp. 1109-1117, 2009.

[9] J. Wu and W. Nyborg, Eds., Emerging therapeutic ultrasound, World Scientific Publishing Co. Pte. Ltd., Hackensack, NJ, USA, 2006.

[10] W. M. Madigosky, I. Rosenbaum, and R. Lucas, "Sound velocities and B/A in fluorocarbon fluids and in several low density solids," Journal of the Acoustical Society of America, vol. 69, no. 6, pp. 1639-1643, 1981.

[11] C. Pantea, D. N. Sinha, C. F. Osterhoudt, and P. C. Mombourquette, "Acoustic nonlinearity in Fluorinert FC-43," in Proceedings of the Meetings on Acoustics (POMA '09), vol. 6, no. 1, pp. 045005-1-045005-14, 2009.

[12] B. T. Sturtevant, C. Pantea, and D. N. Sinha, "The acoustic nonlinearity parameter in Fluorinert up to $381 \mathrm{k}$ and $13.8 \mathrm{MPa}$," Journal of the Acoustical Society of America, vol. 138, no. 1, pp. EL31-EL35, 2015.

[13] J. R. Davies, J. Tapson, and B. J. P. Mortimer, "Novel phase locked cavity resonator for B/A measurements in fluids," Ultrasonics, vol. 38, no. 1-8, pp. 284-291, 2000.

[14] C. K. Vu, D. N. Sinha, C. Pantea, K. Nihei, D. P. Schmitt, and C. Skelt, "Device and Method for Generating a Beam of Acoustic Energy from a Borehole, and Applications Thereof," US Patent 7,839,718, 2010.

[15] C. Vanhille and C. Campos-Pozuelo, "Nonlinear ultrasonic propagation in bubbly liquids: a numerical model," Ultrasound in Medicine and Biology, vol. 34, no. 5, pp. 792-808, 2008.

[16] E. A. Zabolotskaya and S. I. Soluyan, "Emission of harmonic and combination-frequency waves by air bubbles," Soviet PhysicsAcoustics, vol. 18, no. 3, pp. 396-398, 1973.

[17] M. F. Hamilton, Y. A. Il'Inskii, and E. A. Zabolotskaya, "Dispersion," in Nonlinear Acoustics, M. F. Hamilton and D. T. Blackstock, Eds., pp. 151-175, Academic Press, San Diego, Calif, USA, 1998.

[18] S. Saito, "Ultrasound field and bubbles," in Sonochemistry and the Acoustic Bubble, F. Grieser, P. K. Choi, N. Enomoto, H. Harada, K. Okitsu, and K. Yasui, Eds., pp. 11-39, Elsevier, Amsterdam, The Netherlands, 2015.

[19] K. Naugolnykh and L. Ostrovsky, Nonlinear Wave Processes in Acoustics, Cambridge University Press, New York, NY, USA, 1998.

[20] C. Vanhille and C. Campos-Pozuelo, "Simulation of nonlinear ultrasonic pulses propagating through bubbly layers in a liquid: filtering and characterization," Journal of Computational Acoustics, vol. 18, no. 1, pp. 47-68, 2010.

[21] R. T. Beyer, "Parameter of nonlinearity in fluids," Journal of the Acoustical Society of America, vol. 32, no. 6, pp. 719-721, 1960. 
[22] K. D. Wallace, C. W. Lloyd, M. R. Holland, and J. G. Miller, "Finite amplitude measurements of the nonlinear parameter $\mathrm{B} / \mathrm{A}$ for liquid mixtures spanning a range relevant to tissue harmonic mode," Ultrasound in Medicine and Biology, vol. 33, no. 4, pp. 620-629, 2007.

[23] W. K. Law, L. A. Frizzell, and F. Dunn, "Comparison of thermodynamic and finite amplitude methods of B/A measurement in biological materials," Journal of the Acoustical Society of America, vol. 74, no. 4, pp. 1295-1297, 1983.

[24] B. T. Sturtevant, C. Pantea, and D. N. Sinha, "Measured sound speeds and acoustic nonlinearity parameter in liquid water up to $523 \mathrm{~K}$ and $14 \mathrm{MPa}$, AIP Advances, vol. 6, no. 7, Article ID 075310, 2016.

[25] C. Pantea, C. F. Osterhoudt, and D. N. Sinha, "Determination of acoustical nonlinear parameter $\beta$ of water using the finite amplitude method," Ultrasonics, vol. 53, no. 5, pp. 1012-1019, 2013.

[26] R. T. Beyer, “The parameter B/A," in Nonlinear Acoustics, M. F. Hamilton and D. T. Blackstock, Eds., pp. 25-39, Academic Press, San Diego, Calif, USA, 1998.

[27] J.-Y. Kim, L. J. Jacobs, J. Qu, and J. W. Littles, “Experimental characterization of fatigue damage in a nickel-base superalloy using nonlinear ultrasonic waves," Journal of the Acoustical Society of America, vol. 120, no. 3, pp. 1266-1273, 2006.

[28] C. Vanhille, C. Campos-Pozuelo, and D. N. Sinha, "Nonlinear frequency mixing in a resonant cavity: numerical simulations in a bubbly liquid," Ultrasonics, vol. 54, no. 8, pp. 2051-2054, 2014.

[29] C. Vanhille and C. Campos-Pozuelo, "Numerical simulation of nonlinear ultrasonic standing waves in bubbly liquid," International Journal of Nonlinear Sciences and Numerical Simulation, vol. 10, no. 6, pp. 751-757, 2009. 


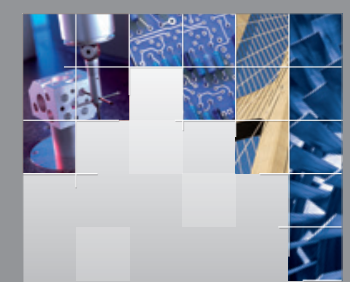

\section{Enfincering}
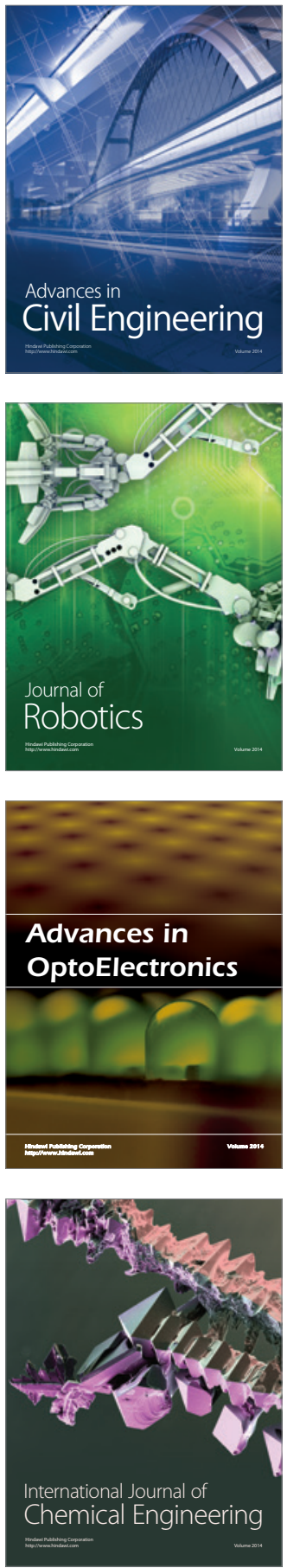

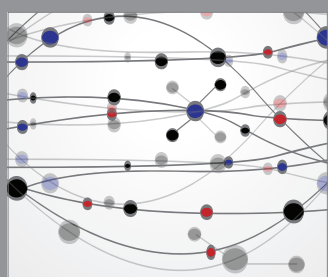

The Scientific World Journal

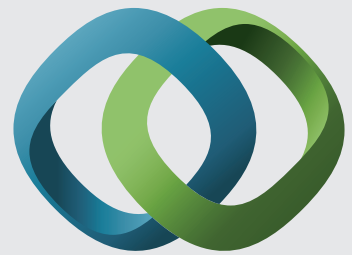

\section{Hindawi}

Submit your manuscripts at

https://www.hindawi.com
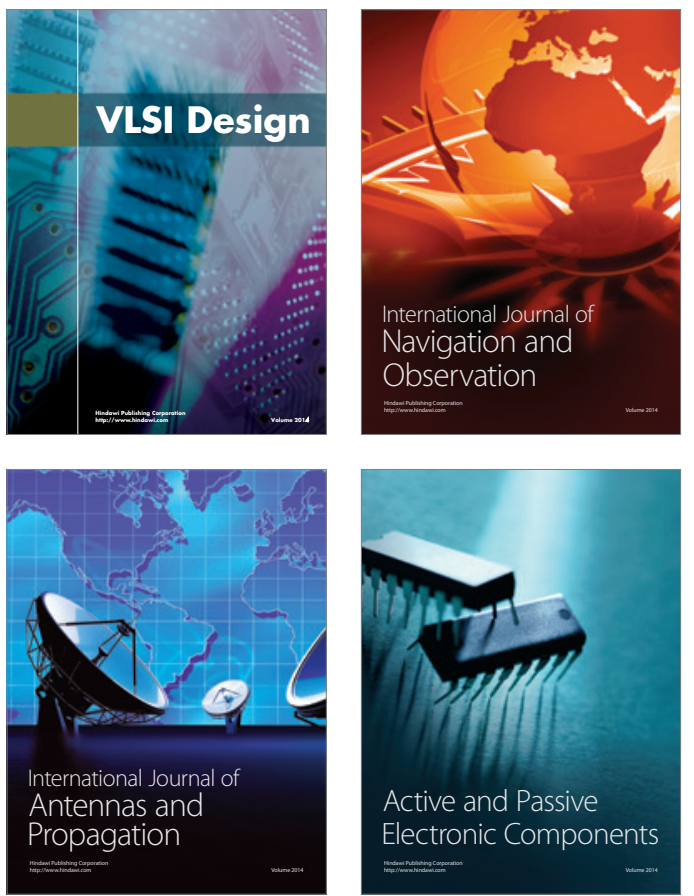
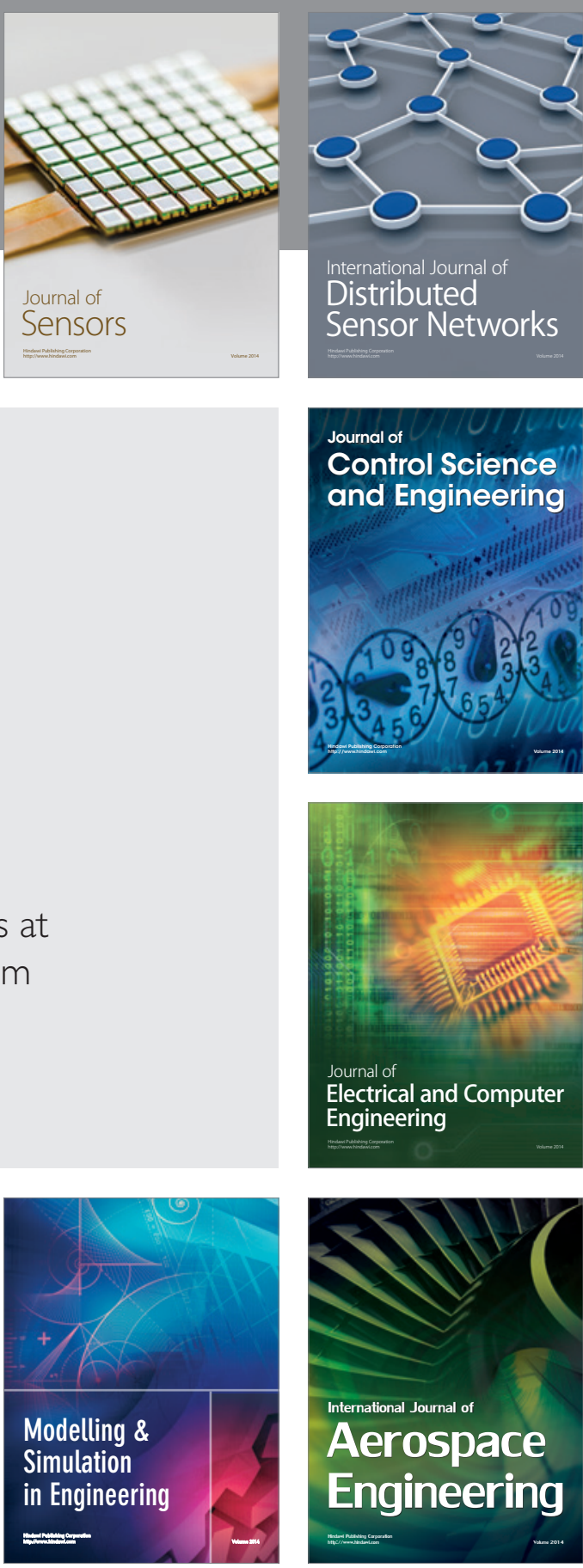

International Journal of

Distributed

Sensor Networks

$-$

Joumal of

Control Science

and Engineering
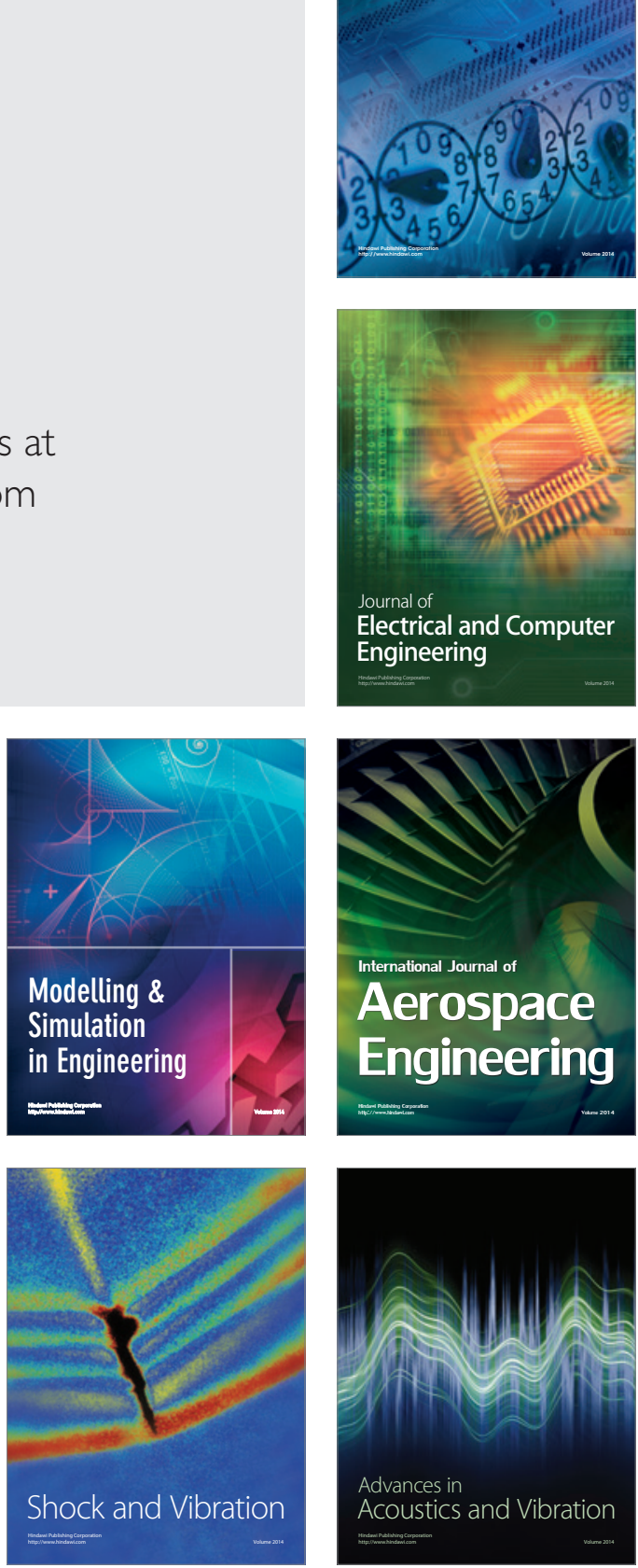